\title{
Axially symmetric particlelike solutions with the flux of a magnetic field in the non-Abelian Proca-Higgs theory
}

\author{
Vladimir Dzhunushaliev $\circledast^{1,2,3, *}$ and Vladimir Folomeev $\circledast^{2,3,4, \uparrow}$ \\ ${ }^{1}$ Department of Theoretical and Nuclear Physics, Al-Farabi Kazakh National University, \\ Almaty 050040, Kazakhstan \\ ${ }^{2}$ Institute of Nuclear Physics, Almaty 050032, Kazakhstan \\ ${ }^{3}$ Academician J. Jeenbaev Institute of Physics of the NAS of the Kyrgyz Republic, \\ 265 a, Chui Street, Bishkek 720071, Kyrgyzstan \\ ${ }^{4}$ International Laboratory for Theoretical Cosmology, \\ Tomsk State University of Control Systems and Radioelectronics (TUSUR), Tomsk 634050, Russia
}

(Received 15 October 2021; accepted 30 November 2021; published 29 December 2021)

\begin{abstract}
Within the non-Abelian SU(2) Proca-Higgs theory, we study localized axially symmetric solutions possessing a finite field energy. It is shown that in a certain sense such solutions are analogs of the NielsenOlesen tube, since they have a longitudinal magnetic field creating a flux of this field over the central cross section of the Proca tube. The main difference between the Proca tube and the Nielsen-Olesen tube is that the Proca tube is described by a topologically trivial solution and has finite size, since its energy density decreases exponentially with distance. The dependence of the total field mass of the Proca tube on the value of one of the parameters determining the solution is examined in detail. The solutions are obtained both in the presence and in the absence of external sources (charge and current densities).
\end{abstract}

DOI: 10.1103/PhysRevD.104.116027

\section{INTRODUCTION}

In recent years interest in systems involving various massive vector fields has increased considerably. Such fields do emerge, in particular, when considering Proca theories. The presence of a mass of vector fields results in substantial differences in the behavior of Proca fields compared with Yang-Mills fields: the Proca fields decay exponentially fast at spatial infinity, which is impossible in principle for the Yang-Mills fields.

At the present time, studies of Proca theories are performed in various directions. In particular, within the past few years, there was prolific activity in the field of physics of Proca stars [1-5] and black holes modeled within generalized Proca theories [6]. The massive extension of a SU(2) gauge theory is studied in Refs. [7,8], the static and spherically symmetric solutions in a class of the generalized Proca theory with the nonminimal coupling to the Einstein tensor are found in Ref. [9], the cosmological

\footnotetext{
*v.dzhunushaliev@gmail.com

†vfolomeev@mail.ru
}

Published by the American Physical Society under the terms of the Creative Commons Attribution 4.0 International license. Further distribution of this work must maintain attribution to the author(s) and the published article's title, journal citation, and DOI. Funded by SCOAP . implications of generalized Proca theories are under investigation in Ref. [10].

In Refs. [11-13], we have studied infinite cylindrically symmetric solutions (tubes) containing a longitudinal electric field. The solutions have been obtained within the non-Abelian Proca-Higgs theory. It is of great interest to get similar solutions describing finite-size objects; this implies that the field energy density should decrease away from the center of a configuration sufficiently fast. In this connection, in the present study we extend the results of Refs. [11-13] and examine axially symmetric solutions in the non-Abelian Proca-Higgs theory with a longitudinal color magnetic field directed along the symmetry axis and transverse electric fields located in a plane perpendicular to the symmetry axis. As will be shown below, the asymptotic behavior of the fields leads to the fact that the total energy of such configurations is finite; this enables us to call such solutions as particlelike solutions. At the same time, the field configurations obtained have a non-Abelian longitudinal magnetic Proca field; this enables us to call them tubes with a flux of the corresponding magnetic field over a plane perpendicular to the tube axis and located at the center of the axis. The latter means that such a tube can be regarded as an analogue of the Nielsen-Olesen tube [14], since in both cases there is the flux of the magnetic field over the transverse cross section of the tube. On the other hand, there also exist substantial differences: (i) the Nielsen-Olesen tube is a topologically nontrivial configuration, whereas our field configuration is topologically 
trivial; (ii) the Proca tube has finite size, since the fields decrease exponentially with distance; and (iii) the NielsenOlesen tube contains an Abelian magnetic field, whereas the Proca tube-a non-Abelian magnetic field.

It should be also noted that in our study we consider a system supported by Proca fields together with a Higgs scalar field. The presence of the latter, as will be shown below, leads to a very interesting result: it initiates the process where the Proca fields are being pushed outside by the Higgs field. This interesting result is an analogue of the Meissner phenomenon in superconductivity and of the dual Meissner effect in QCD.

The field configurations under investigation can emerge as quasiparticles in the hypothetical "quark-Proca-gluonHiggs" plasma. In this connection we note that, in QCD, it is assumed that in the quark-gluon plasma there exist multiple bound states of quasiparticles (for details see Ref. [15] where the existence of such quasiparticles has been suggested, as well as Ref. [16] where studies in this direction have been continued and the role of multiple (colored) bound states in the quark-gluon plasma phase at not too high temperature is demonstrated).

Thus, we will study here particlelike solutions in the Proca-Higgs theory in which there is a color longitudinal Proca field directed along the symmetry axis; this enables us to call such solutions as Proca tubes with a flux of the magnetic field. The paper is organized as follows. In Sec. II, we write down the general field equations for the nonAbelian-Proca-Higgs theory. In Sec. III, we obtain cylindrically symmetric solutions to the equations of Sec. II describing infinite tubes with the flux of the longitudinal color magnetic field. In Sec. IV, we find axially symmetric solutions to the equations of Sec. II (both with and without charge and current densities) describing finite-size tubes with the flux of the longitudinal chromomagnetic field. Finally, in Sec. V, we summarize and discuss the results obtained in the present paper.

\section{NON-ABELIAN SU(3) PROCA-HIGGS THEORY}

The Lagrangian describing a system consisting of a nonAbelian SU(3) Proca field $A_{\mu}^{a}$ interacting with nonlinear scalar field $\phi$ can be taken in the form (hereafter, we work in units such that $c=\hbar=1$ )

$$
\begin{aligned}
\mathcal{L}= & -\frac{1}{4} F_{\mu \nu}^{a} F^{a \mu \nu}-\frac{1}{2}\left(\mu^{2}\right)^{a b, \mu}{ }_{\nu} A_{\mu}^{a} A^{b \nu}+\frac{1}{2} \partial_{\mu} \phi \partial^{\mu} \phi \\
& +\frac{\lambda}{2} \phi^{2} A_{\mu}^{a} A^{a \mu}-\frac{\Lambda}{4}\left(\phi^{2}-M^{2}\right)^{2} .
\end{aligned}
$$

Here $F_{\mu \nu}^{a}=\partial_{\mu} A_{\nu}^{a}-\partial_{\nu} A_{\mu}^{a}+g f_{a b c} A_{\mu}^{b} A_{\nu}^{c}$ is the field strength tensor for the Proca field, where $f_{a b c}$ are the SU(3) structure constants, $g$ is the coupling constant, $a, b, c=$ $1,2, \ldots, 8$ are color indices, $\mu, \nu=0,1,2,3$ are spacetime indices. The Lagrangian (1) also contains the arbitrary constants $M, \lambda, \Lambda$ and the Proca field mass tensor $\left(\mu^{2}\right)^{a b, \mu}{ }_{\nu}$.
Using (1), the corresponding field equations can be written in the form

$$
\begin{gathered}
D_{\nu} F^{a \mu \nu}-\lambda \phi^{2} A^{a \mu}=-\left(\mu^{2}\right)^{a b, \mu}{ }_{\nu} A^{b \nu}+j^{a \mu}, \\
\square \phi=\lambda A_{\mu}^{a} A^{a \mu} \phi+\Lambda \phi\left(M^{2}-\phi^{2}\right),
\end{gathered}
$$

where, for the sake of generality, we have also added the current four-vector $j^{a \mu}$. For such systems, the field energy density is

$$
\begin{aligned}
\varepsilon= & \frac{1}{2}\left(E_{i}^{a}\right)^{2}+\frac{1}{2}\left(H_{i}^{a}\right)^{2}-\left(\mu^{2}\right)^{a b, \alpha}{ }_{0} A_{\alpha}^{a} A_{0}^{b}+\frac{1}{2}\left(\mu^{2}\right)^{a b, \alpha}{ }_{\beta} A_{\alpha}^{a} A^{b \beta} \\
& +\frac{1}{2}\left(\partial_{t} \phi\right)^{2}+\frac{1}{2}(\nabla \phi)^{2}+\lambda \phi^{2}\left[\left(A_{0}^{a}\right)^{2}-\frac{1}{2} A_{\alpha}^{a} A^{a \alpha}\right] \\
& +\frac{\Lambda}{4}\left(\phi^{2}-M^{2}\right)^{2}
\end{aligned}
$$

where $i=1,2,3$ and $E_{i}^{a}$ and $H_{i}^{a}$ are the components of the electric and magnetic field strengths, respectively.

In the present paper we will consider solutions belonging to the subgroup $\mathrm{SU}(2) \subset \mathrm{SU}(3)$ spanned on the Gell-Mann matrices $\lambda^{2,5,7}$.

\section{INFINITE FLUX TUBE SOLUTIONS}

To begin with, it will be helpful to study a simpler system with an infinite flux tube containing a flux of a longitudinal electric field [11-13]. To describe such a tube, let us choose the ansatz for the field potentials in the form

$$
A_{t}^{2}=\frac{f(\rho)}{g}, \quad A_{\varphi}^{7}=\frac{\rho w(\rho)}{g}, \quad \phi=\phi(\rho)
$$

written in cylindrical coordinates $\{t, \rho, z, \varphi\}$. Such a tube contains the following color electric and magnetic fields (physical components):

$$
E_{\rho}^{2}=-\frac{f^{\prime}}{g}, \quad E_{\varphi}^{5}=-\frac{f w}{2 g}, \quad H_{z}^{7}=-\frac{\rho w^{\prime}+w}{g \rho} .
$$

(Henceforth in this section the prime denotes differentiation with respect to $\rho$.) In this case the energy flux is absent, since all components of the Poynting vector are zero,

$$
S^{i}=\frac{\epsilon^{i j k}}{\sqrt{\gamma}} E_{j}^{a} H_{k}^{a}=0
$$

where $\epsilon^{i j k}$ is the completely antisymmetric Levi-Civita symbol and $\gamma$ is the determinant of the space metric.

Substituting the potentials (5) in Eqs. (2) and (3), we get the following set of equations (without the current):

$$
f^{\prime \prime}+\frac{f^{\prime}}{\rho}=f\left(\frac{w^{2}}{4}+\lambda \phi^{2}-\mu_{1}^{2}\right),
$$




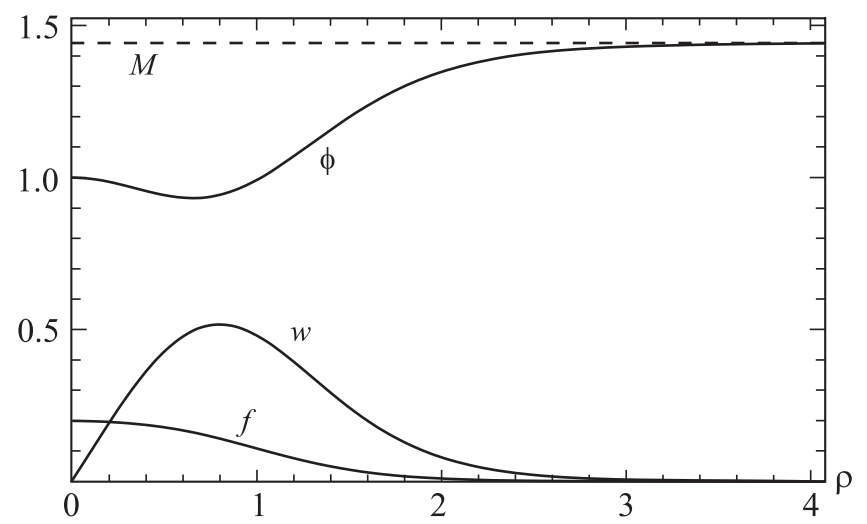

FIG. 1. The graphs of the Proca field potentials $f, w$ and of the scalar field $\phi$ for the following values of the system parameters: $\lambda=10, \Lambda=1, g=1, f_{0}=0.2, \phi_{0}=1, w_{1}=1, M=1.44227$, $\mu_{1}=3.359585, \mu_{2}=3.7633$.

$$
\begin{gathered}
w^{\prime \prime}+\frac{w^{\prime}}{\rho}-\frac{w}{\rho^{2}}=w\left(-\frac{f^{2}}{4}+\lambda \phi^{2}-\mu_{2}^{2}\right), \\
\phi^{\prime \prime}+\frac{\phi^{\prime}}{\rho}=\phi\left[\frac{\lambda}{g^{2}}\left(-f^{2}+w^{2}\right)+\Lambda\left(\phi^{2}-M^{2}\right)\right]
\end{gathered}
$$

with the following components of the Proca field mass tensor: $\mu_{1}^{2}=\left(\mu^{2}\right)^{22, t}{ }_{t}$ and $\mu_{2}^{2}=\left(\mu^{2}\right)^{77, \varphi}{ }_{\varphi}$. We seek a solution to Eqs. (8)-(10) in the vicinity of the origin of coordinates in the form

$$
\begin{aligned}
f(\rho) & =f_{0}+f_{2} \frac{\rho^{2}}{2}+\ldots \quad \text { with } f_{2}=\frac{f_{0}}{2}\left[\lambda \phi_{0}^{2}-\mu_{1}^{2}\right], \\
w(\rho) & =w_{1} \rho+\ldots, \\
\phi(\rho) & =\phi_{0}+\phi_{2} \frac{\rho^{2}}{2}+\ldots \quad \text { with } \\
\phi_{2} & =\frac{\phi_{0}}{2 g^{2}}\left[-\lambda f_{0}^{2}+g^{2} \Lambda\left(\phi_{0}^{2}-M^{2}\right)\right],
\end{aligned}
$$

where the expansion coefficients $f_{0}, \phi_{0}$, and $w_{1}$ are arbitrary.

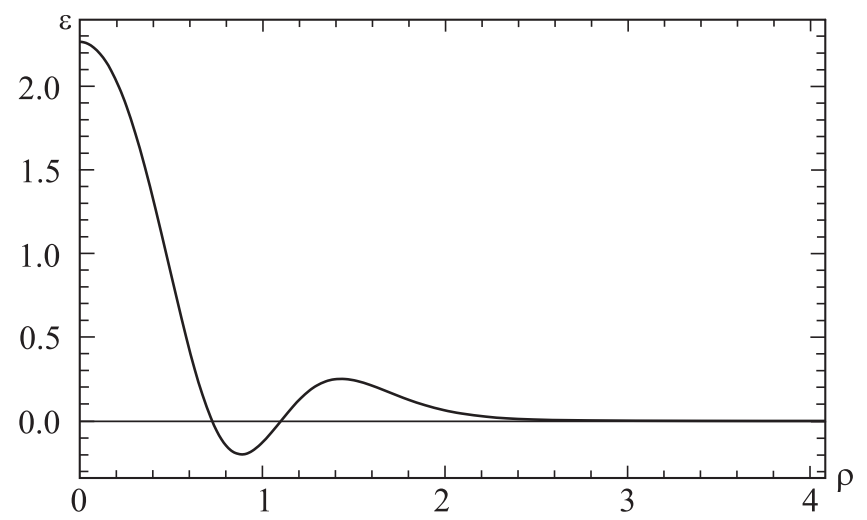

FIG. 2. The profile of the flux tube energy density from Eq. (11) for the solutions given in Fig. 1.

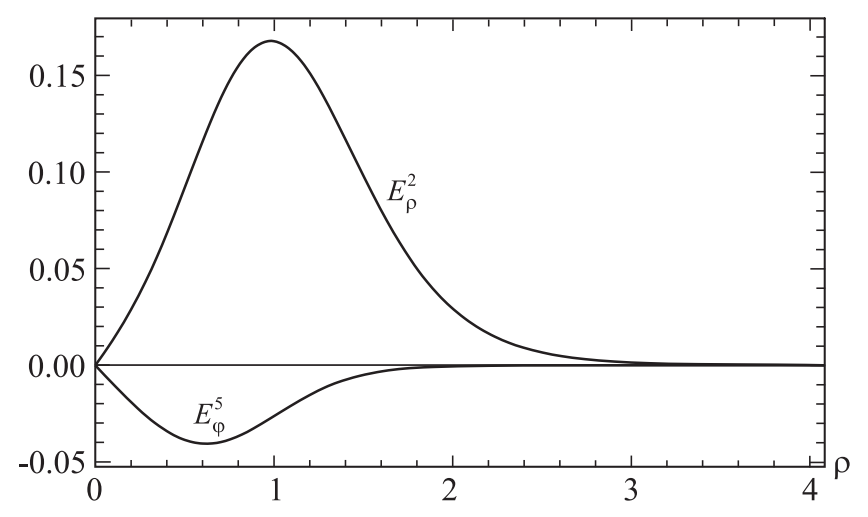

FIG. 3. The profiles of the color electric fields $E_{\rho}^{2}$ and $E_{\varphi}^{5}$ from Eq. (6) for the solutions given in Fig. 1.

The derivation of solutions to the set of equations (8)(10) is an eigenvalue problem for the parameters $\mu_{1}, \mu_{2}$, and $M$. The numerical solution describing the behavior of the Proca field potentials and of the corresponding electric and magnetic fields is given in Figs. 1-4. In particular, Fig. 2 shows the energy density obtained from Eq. (4) using (5) and (6) in the form

$$
\begin{aligned}
\varepsilon= & \frac{1}{g^{2}}\left[\frac{f^{\prime 2}}{2}+\frac{w w^{\prime}}{\rho}+\frac{w^{\prime 2}}{2}+\frac{g^{2}}{2} \phi^{\prime 2}+\frac{w^{2}}{2 \rho^{2}}+\frac{f^{2} w^{2}}{8}-\frac{\mu_{1}^{2} f^{2}}{2}\right. \\
& \left.-\frac{\mu_{2}^{2} w^{2}}{2}+\frac{\lambda}{2}\left(f^{2}+w^{2}\right) \phi^{2}+\frac{g^{2} \Lambda}{4}\left(\phi^{2}-M^{2}\right)^{2}\right] .
\end{aligned}
$$

The asymptotic behavior of the functions $f, w$, and $\phi$, which follows from Eqs. (8)-(10), is

$$
\begin{aligned}
f(\rho) & \approx f_{\infty} \frac{e^{-\rho \sqrt{\lambda M^{2}-\mu_{1}^{2}}}}{\sqrt{\rho}}, \quad w(\rho) \approx w_{\infty} \frac{e^{-\rho \sqrt{\lambda M^{2}-\mu_{2}^{2}}}}{\sqrt{\rho}}, \\
\phi & \approx M-\phi_{\infty} \frac{e^{-\rho \sqrt{2 \Lambda M^{2}}}}{\sqrt{\rho}},
\end{aligned}
$$

where $f_{\infty}, w_{\infty}$, and $\phi_{\infty}$ are integration constants.

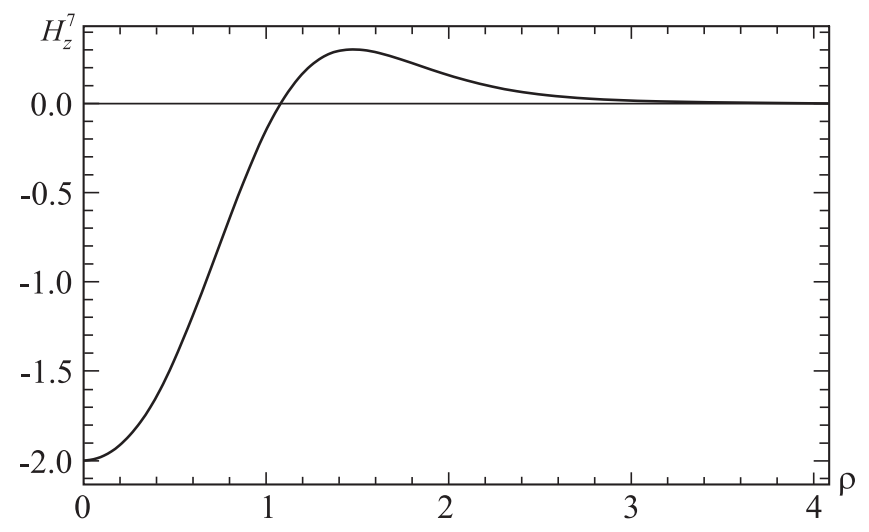

FIG. 4. The profile of the color magnetic field $H_{z}^{7}$ from Eq. (6) for the solutions given in Fig. 1. 
It is seen from Figs. 1-4 that the cylindrically symmetric solutions found can be used to describe infinitely long tubes within the non-Abelian Proca-Higgs theory under consideration. Such tubes possess the finite linear energy density (see Fig. 2),

$$
\mathcal{E}=2 \pi \int_{0}^{\infty} \rho \varepsilon(\rho) d \rho<\infty,
$$

and the finite flux of the longitudinal color magnetic field $H_{z}^{7}$ (see Fig. 4),

$$
\Phi_{z}^{H}=2 \pi \int_{0}^{\infty} \rho H_{z}^{7} d \rho<\infty .
$$

Note that, unlike the systems considered by us earlier in Refs. [11-13], these tubes contain neither the flux of the longitudinal color electric field $E_{z}^{a}$ nor the energy flux/ momentum density (since the Poynting vector is zero).

\section{FINITE FLUX TUBE SOLUTIONS}

Let us now extend the solutions of the previous section to the case of finite-size configurations. To obtain static axially symmetric solutions, we employ the ansatz (5), with an obvious generalization to the case where the components of the four-potential depend also on the coordinate $z$. As will be demonstrated below, in this case, it is possible to get solutions describing configurations possessing finite sizes both along the $\rho$-axis and along the $z$-axis.

For convenience of performing numerical computations, we employ spherical coordinates $\{t, r, \theta, \varphi\}$ in which the ansatz (5) can be recast in the form

$A_{t}^{2}=\frac{f(r, \theta)}{g}, \quad A_{\varphi}^{7}=r \sin \theta \frac{w(r, \theta)}{g}, \quad \phi=\phi(r, \theta)$,

where all functions now depend on $r$ and $\theta$. For such ansatz, there are the following nonzero physical components of color electric and magnetic fields:

$$
\begin{gathered}
E_{r}^{2}=-\frac{f_{, r}}{g}, \quad E_{\theta}^{2}=-\frac{f_{, \theta}}{g r}, \quad E_{\varphi}^{5}=-\frac{f w}{2 g}, \\
H_{r}^{7}=-\frac{w_{, \theta}+w \cot \theta}{g r}, \quad H_{\theta}^{7}=\frac{w+r w_{, r}}{g r} .
\end{gathered}
$$

(Henceforth a comma in lower indices denotes differentiation with respect to the corresponding coordinate.) In turn, the field equations (2) and (3) yield

$$
f_{, r r}+\frac{2}{r} f_{, r}+\frac{1}{r^{2}}\left(f_{, \theta \theta}+\cot \theta f_{, \theta}\right)-\frac{1}{4} f\left(w^{2}+4 \lambda \phi^{2}\right)+\mu_{1}^{2} f=j^{2 t},
$$

$$
\begin{gathered}
w_{, r r}+\frac{2}{r} w_{, r}+\frac{1}{r^{2}}\left(w_{, \theta \theta}+\cot \theta w_{, \theta}\right)-\frac{w}{r^{2} \sin ^{2} \theta} \\
+\frac{1}{4}\left(f^{2}-4 \lambda \phi^{2}\right) w+\mu_{2}^{2} w=-r \sin \theta j^{7 \varphi}, \\
\phi_{, r r}+\frac{2}{r} \phi_{, r}+\frac{1}{r^{2}}\left(\phi_{, \theta \theta}+\cot \theta \phi_{, \theta}\right)+\frac{\lambda}{g^{2}}\left(f^{2}-w^{2}\right) \phi \\
-\Lambda \phi\left(\phi^{2}-M^{2}\right)=0 .
\end{gathered}
$$

The results of numerical simulations for these equations are given in Sec. IV A (in the absence of the currents) and in Sec. IV B (in the presence of the currents).

\section{A. The case with no charges}

Consider first the case where the currents $j^{a \mu}=0$ in Eqs. (15) and (16). To solve the set of equations (15)-(17), it is necessary to impose appropriate boundary conditions for the Proca and scalar fields at the origin $(r=0)$, at infinity $(r \rightarrow \infty)$, on the positive $z$ axis $(\theta=0)$, and, using the reflection symmetry with respect to $\theta \rightarrow \pi-\theta$, in the $\{x, y\}$ plane $(\theta=\pi / 2)$. So we require

$$
\begin{aligned}
\left.\frac{\partial f}{\partial r}\right|_{r=0} & =\left.\frac{\partial \phi}{\partial r}\right|_{r=0}=0,\left.\quad w\right|_{r=0}=0 \\
\left.f\right|_{r=\infty} & =0,\left.\quad w\right|_{r=\infty}=0,\left.\quad \phi\right|_{r=\infty}=M ; \\
\left.\frac{\partial f}{\partial \theta}\right|_{\theta=0} & =\left.\frac{\partial \phi}{\partial \theta}\right|_{\theta=0}=0,\left.\quad w\right|_{\theta=0}=0 ; \\
\left.\frac{\partial f}{\partial \theta}\right|_{\theta=\pi / 2} & =\left.\frac{\partial w}{\partial \theta}\right|_{\theta=\pi / 2}=\left.\frac{\partial \phi}{\partial \theta}\right|_{\theta=\pi / 2}=0
\end{aligned}
$$

The set of three coupled nonlinear elliptic partial differential equations (15)-(17) has been solved numerically subject to the above boundary conditions. Calculations are carried out using the package FIDISOL [17] where the numerical method based on the NewtonRaphson method is employed. This method provides an iterative procedure for obtaining an exact solution starting from an approximate solution (an initial guess). The essence of the procedure is that, in the first step, a correction is computed that improves the approximate solution. Then this improved approximate solution is used in the next step to obtain the next correction that gives a better approximation to the exact solution. Repeating the calculations iteratively, the approximate solution will eventually converge to the exact solution to a prescribed accuracy.

To obtain a correct numerical solution, there are two key points. First, it is essential to have a good initial guess for starting the iteration procedure. As such initial guess, we used the solution for the infinite tube obtained in Sec. III. Second, an important point is the accuracy of numerical solutions being obtained. The accuracy is directly related to the discretization of the set of partial differential equations: 
it depends on the number of grid points and on the order of consistency of the differential formulas for the derivatives. Typical grids used by us have sizes $201 \times 51$ points, covering the region of integration in the $r$ and $\theta$ directions, respectively. This enabled us to get solutions with typical relative errors less than $10^{-4}$. $\mu_{2}=3.7$
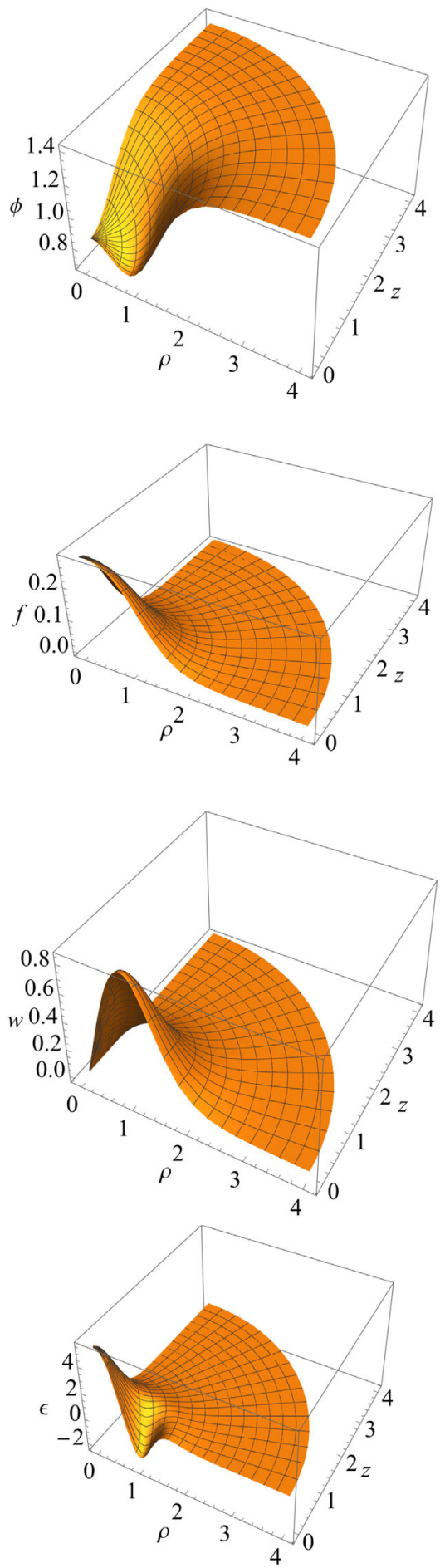

$\mu_{2}=2.5$
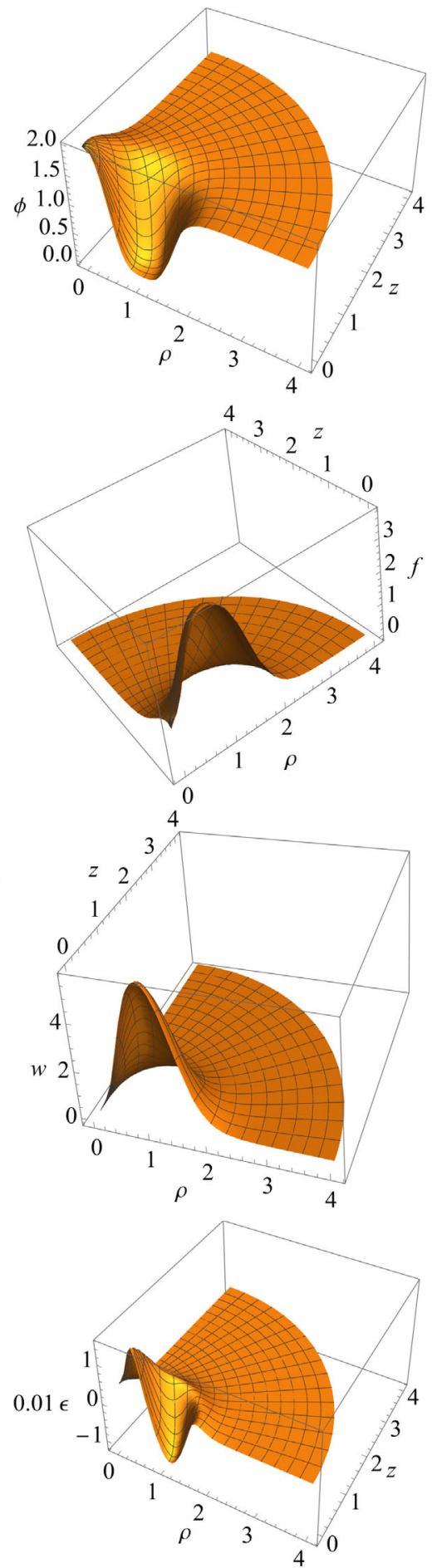

$\mu_{2}=1.02$
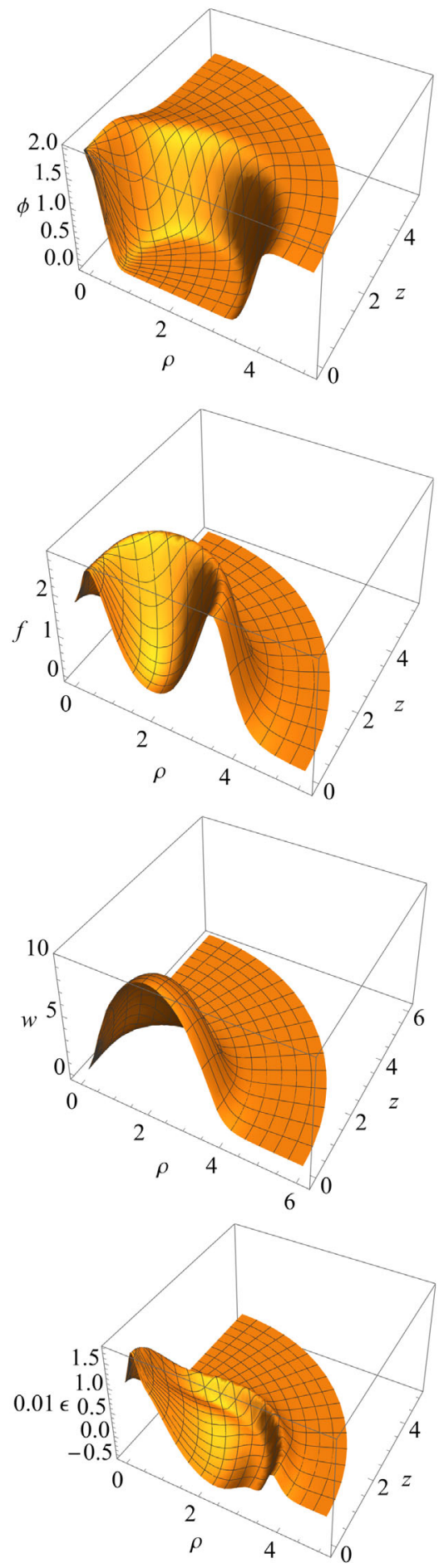

FIG. 5. Distributions of the fields $\phi$ (upper row), $f$ (second row), $w$ (third row), and the total energy density of the system $\varepsilon$ from Eq. (18) (lower row) for different values of $\mu_{2}$ and fixed values of the parameters $M=1.4, \Lambda=1, \lambda=10, g=1$, and $\mu_{1}=3.3$ are shown as functions of the coordinates $\rho=r \sin \theta$ and $z=r \cos \theta$. 
As in the case of an infinite tube of Sec. III, the input parameters are eigenparameters $\mu_{1}, \mu_{2}$, and $M$, whose magnitudes determine the solution completely. (Notice here that by imposing appropriate boundary conditions to Eqs. (15)-(17), one can reproduce solutions for an infinite flux tube obtained in Sec. III.) For different values of the aforementioned parameters, one can obtain qualitatively different field distributions, some typical examples of which are depicted in Fig. 5.

In particular, Fig. 5 shows the total energy density of the system under consideration obtained from (4) using (12)-(14) in the form

$$
\begin{aligned}
\varepsilon= & \frac{1}{g^{2}}\left[\frac{f_{, \theta}^{2}}{2 r^{2}}+\frac{f_{, r}^{2}}{2}+\frac{w_{, r}^{2}}{2}+\frac{w_{, \theta}^{2}}{2 r^{2}}+\cot \theta \frac{w w_{, \theta}}{r^{2}}+\frac{w w_{, r}}{r}\right. \\
& +\frac{g^{2}}{2}\left(\frac{\phi_{, \theta}^{2}}{r^{2}}+\phi_{, r}^{2}\right)+\frac{f^{2} w^{2}}{8}-\frac{\mu_{1}^{2}}{2} f^{2}-\frac{\mu_{2}^{2}}{2} w^{2} \\
& \left.+\frac{\lambda}{2} \phi^{2}\left(f^{2}+w^{2}\right)+\csc ^{2} \theta \frac{w^{2}}{2 r^{2}}+\frac{g^{2} \Lambda}{4}\left(\phi^{2}-M^{2}\right)^{2}\right] .
\end{aligned}
$$

It is seen from the graphs of Fig. 5 that the energy density is negative in some regions. To see whether this will give a negative total energy of the system or not, we calculate the total mass (energy) of the configurations under consideration,

$$
M_{\mathrm{tot}}=2 \pi \int \varepsilon r^{2} \sin \theta d r d \theta
$$

The results of calculations are given in Fig. 6 for the range $1.02 \leq \mu_{2} \leq 4.2$ for fixed values of other system parameters. For $\mu_{2}=\mu_{2 \text { (crit) }} \approx 3.73$, the function $f \rightarrow 0$, and with further increase of $\mu_{2}$, it always remains equal to zero. Correspondingly, the configurations with $\mu_{2} \lesssim \mu_{2 \text { (crit) }}$ possess both the magnetic and electric fields, but for $\mu_{2} \gtrsim$ $\mu_{2 \text { (crit) }}$ only the magnetic field is present. Taking into account that the asymptotic expansions of the solutions at large $r$ give the restriction on the mass $\mu_{2}<\sqrt{\lambda M^{2}}$ (see below), the numerical calculations indicate that when $\mu_{2}$ goes to this upper limit the functions $w \rightarrow 0$ (the magnetic field is switched off) and $\phi \rightarrow M$ over all space. Correspondingly, the energy density and the total mass of the system vanish, as is seen from Fig. 6.

Using the solutions obtained, in Figs. 7-9, we have plotted the distributions of the color electric and magnetic fields for different values of $\mu_{2}$. The analysis of these distributions indicates that

(i) According to Fig. 7, there is a point lying on a circle in the $z=0$ plane where the lines of force of the electric field $\vec{E}^{2}$ penetrate into. Aside from this, for sufficiently small values of $\mu_{2}$, there is a surface in the form of a torus where the lines of force of the electric field penetrate into as well.

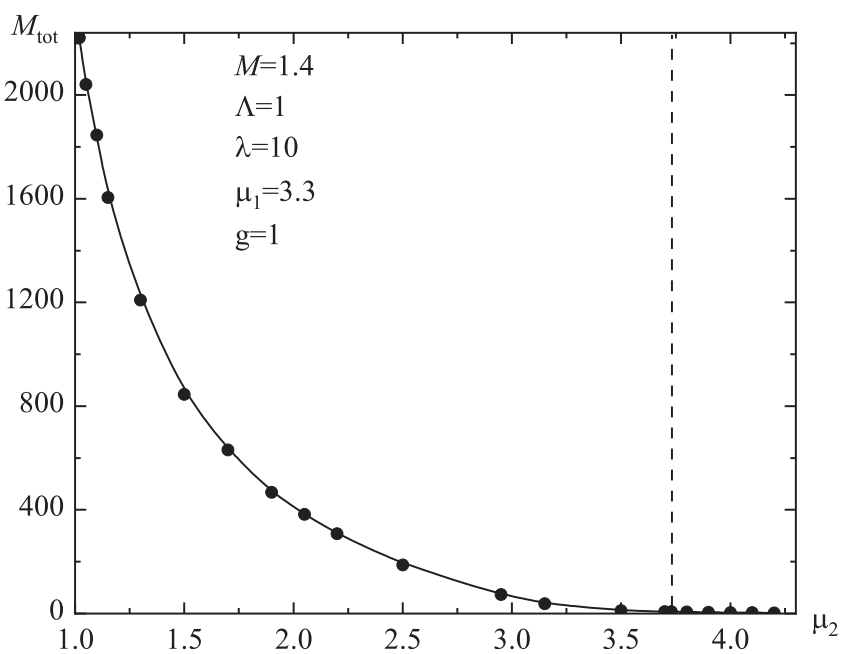

FIG. 6. The dependence of the total mass (19) of the configurations under consideration on $\mu_{2}$ for fixed values of the parameters $M=1.4, \Lambda=1, \lambda=10, g=1$, and $\mu_{1}=3.3$. The vertical dashed line separates the systems containing the electric and magnetic fields (located to the left of the line) from the configurations possessing only the magnetic field (located to the right of the line).

(ii) As is seen from Fig. 9, the magnetic field $\vec{H}^{7}$ possesses a vortex centred at a circle in the $z=0$ plane. In this case, as $\rho \rightarrow 0$, the component $H_{\rho}^{7} \rightarrow 0$, whereas the component $H_{z}^{7}$ remains nonzero; as a result, there is a flux of this field perpendicular to the transverse cross section of the tube.

(iii) The configurations obtained for $\mu_{2} \gtrsim \mu_{2 \text { (crit) }}$ resemble somewhat the Nielsen-Olesen tube: both types of systems contain only a magnetic field with the flux directed along the $z$-axis.

(iv) For all the configurations obtained, the electric field $\vec{E}^{5}$ is a vortex field (see Fig. 8).

Thus, as in the case with an infinite tube of Sec. III, the finite tube under consideration does not contain the flux of the longitudinal chromoelectric field $E_{z}^{2}$,

$$
\Phi_{z}^{E}=2 \pi \int_{0}^{\infty} \rho E_{z}^{2}(z=0, \rho) d \rho=0,
$$

but possesses the flux of the longitudinal chromomagnetic field $H_{z}^{7}$,

$$
\Phi_{z}^{H}=2 \pi \int_{0}^{\infty} \rho H_{z}^{7}(z=0, \rho) d \rho \neq 0 .
$$

Also, the finite tube contains no energy flux density-the Poynting vector (7) is zero. 

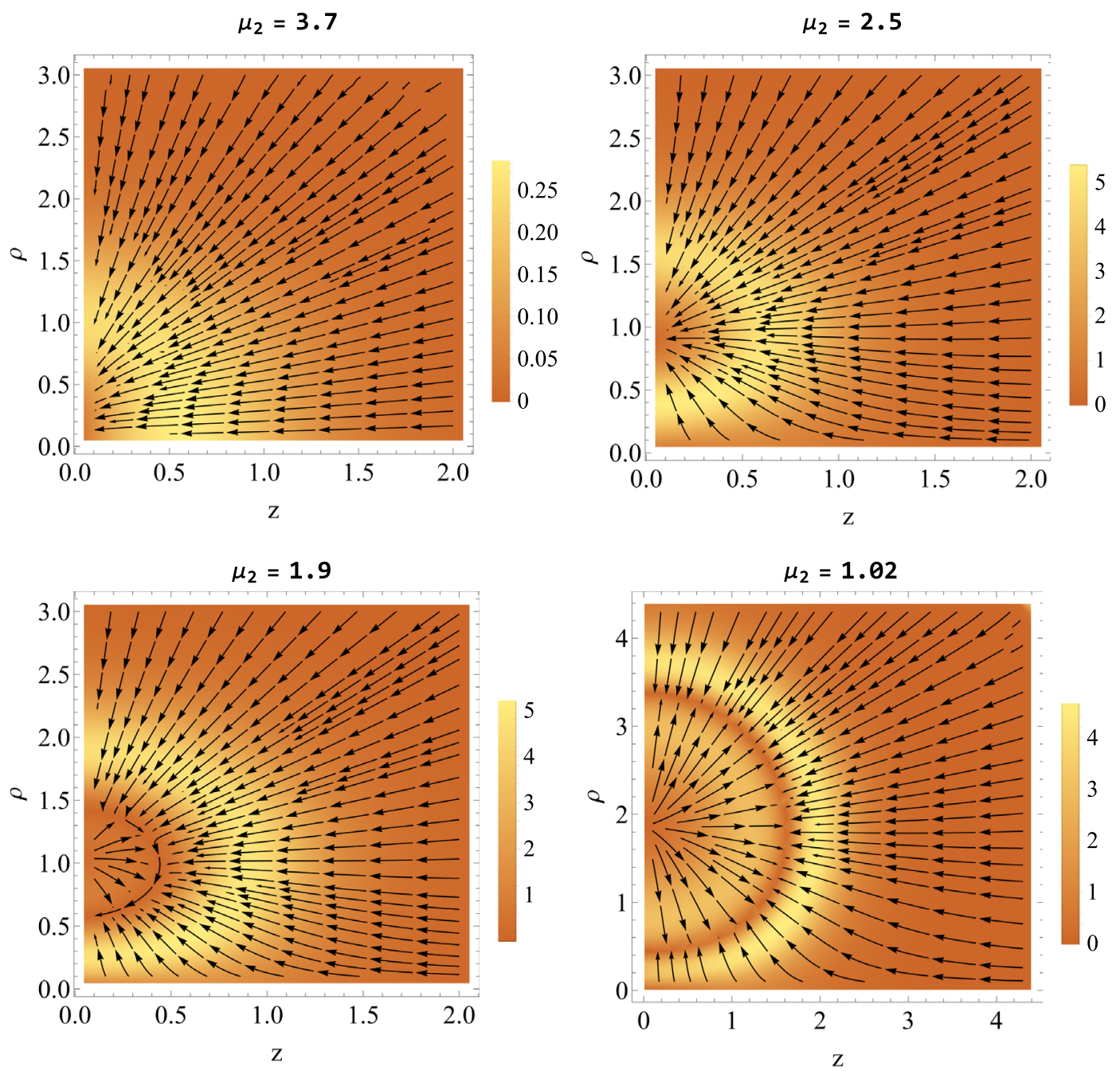

FIG. 7. The color electric field $\vec{E}^{2}$ strength distributions for different values of the Proca mass $\mu_{2}$ and a fixed value $\mu_{1}=3.3$. The parameters $M=1.4, \Lambda=1, \lambda=10, g=1$.

Consider now the asymptotic behavior of the solutions found. As $r \rightarrow \infty$, the fields $f, w \rightarrow 0$ exponentially fast; hence, one can neglect the nonlinear terms in Eqs. (15)-(17) containing $f^{2}$ and $w^{2}$. In turn, asymptotically, the field $\phi \approx M-\eta \rightarrow M$, and the function $\eta$ decays exponentially; this permits us to replace the term $\phi^{2}$ by $M^{2}$ in Eqs. (15) and (16). As a result, from Eqs. (15)-(17), one can obtain the following asymptotic equations:

$$
\begin{gathered}
\triangle_{r, \theta} f+\left(\mu_{1}^{2}-\lambda M^{2}\right) f=0, \\
\triangle_{r, \theta} w-\frac{w}{r^{2} \sin ^{2} \theta}+\left(\mu_{2}^{2}-\lambda M^{2}\right) w=0, \\
\triangle_{r, \theta} \eta-2 \Lambda M^{2} \eta=0,
\end{gathered}
$$

where $\triangle_{r, \theta}$ is the Laplacian operator. Equations (20) and (22) have obvious solutions in the form

$$
\begin{gathered}
f \approx C_{f}(Y)_{l_{f}}^{0} \frac{e^{-r \sqrt{\lambda M^{2}-\mu_{1}^{2}}}}{r}, \\
\eta \approx C_{\eta}(Y)_{l_{\eta}}^{0} \frac{e^{-r \sqrt{2 \Lambda M^{2}}}}{r}
\end{gathered}
$$

where $(Y)_{l_{f, \eta}}^{0}$ are spherical functions and $C_{f, \eta}$ are constants. In turn, Eq. (21) has a solution similar to (23), but only with the angular part expressed in terms of special functions (we do not show this expression here to avoid overburdening the text). It follows from the above expressions that there are upper limits for the masses $\mu_{1}$ and $\mu_{2}$ ensuring the exponential asymptotic decay of the solutions: $\mu_{1}^{2}, \mu_{2}^{2}<\lambda M^{2}$.

\section{B. The case with nonzero charges}

In this subsection we consider the case where the righthand sides of the Proca equations (15) and (16) contain 



FIG. 8. The color electric field $\vec{E}^{5}$ strength distributions for different values of the Proca mass $\mu_{2}$ and a fixed value $\mu_{1}=3.3$. The parameters $M=1.4, \Lambda=1, \lambda=10, g=1$ (the graphs are plotted in the $\{x, y\}$ plane, i.e., when $\theta=\pi / 2$ ).

nonzero current densities. When considering a selfconsistent problem with a tube connecting quarks such currents must be created by spinor fields describing quarks. For the sake of simplicity, here we consider a toy model where the currents are given by hand. This means that the location of the quarks and the magnitude of color currents and charges created by them are fixed.

In the simplest case the currents can be given, for instance, by the Gaussian distribution,

$$
j^{a \mu}=\left(j_{0}\right)^{a \mu} e^{-\frac{R^{2}}{R_{0}^{2}}}
$$

where $\left(j_{0}\right)^{a \mu}$ is an arbitrary constant and $R$ is some function of $z$ and $\rho$.

\section{The case of nonzero charge density $j^{2 t}$}

Consider first the case where there is only the charge density $j^{2 t}$ in Eq. (15). For this case, we choose
$R^{2}=(z-l)^{2}+\rho^{2}$. Such a choice implies that a "quark" is located on the tube axis at a distance $l$ from the origin of coordinates. Choosing different values of the constant $\left(j_{0}\right)^{2 t}$, in Fig. 10, we have plotted the corresponding distributions of the color electric field $\vec{E}^{2}$. As is seen from the figure, as $\left(j_{0}\right)^{2 t}$ increases (in modulus), the behavior of the lines of force changes qualitatively: if for small $\left(j_{0}\right)^{2 t}$ the electric field created by the Proca field by itself is still comparable to the magnitude of the field determined by the charge, it ceases to be so with increase of $\left(j_{0}\right)^{2 t}$ when the field determined by the charge becomes dominating; this results in the aforementioned qualitative changes of the structure of the electric field. This is illustrated by the appearance of a "fixed" point in the $z=0$ plane and at some distance from the tube axis. The behavior of the lines of force is similar here to the behavior of phase trajectories for autonomous differential equations.

Distributions of the electric, $\vec{E}^{5}$, and magnetic, $\vec{H}^{7}$, fields change qualitatively only slightly compared with the case 

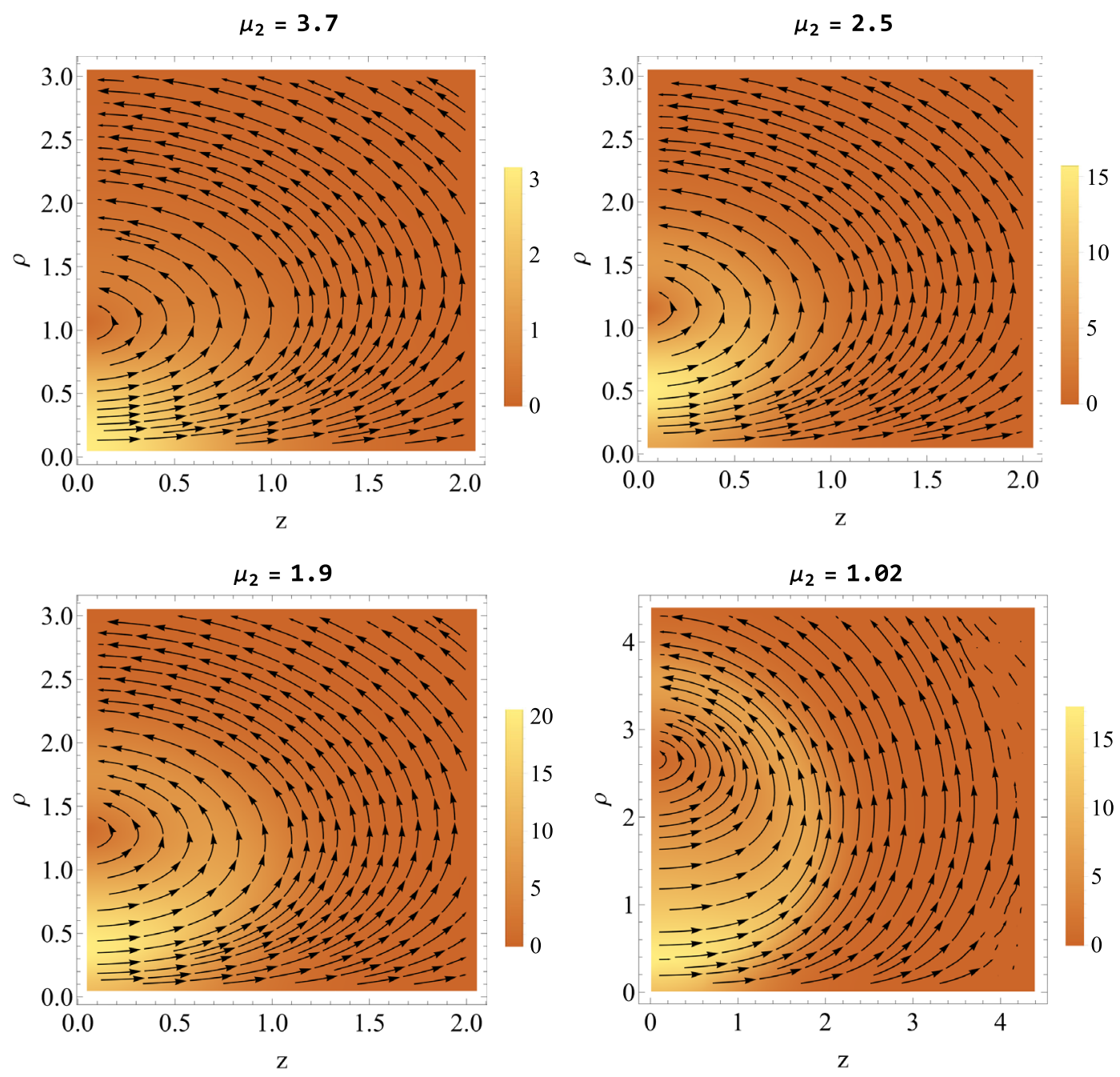

FIG. 9. The color magnetic field $\vec{H}^{7}$ strength distributions for different values of the Proca mass $\mu_{2}$ and a fixed value $\mu_{1}=3.3$. The parameters $M=1.4, \Lambda=1, \lambda=10, g=1$.

without charge. Hence, to avoid overburdening the text, we do not show them here.

\section{The case of nonzero current density $j^{7 \varphi}$}

Consider now the case where there is only the current density $j^{7 \varphi}$ in Eq. (16). For this case, we take $R^{2}=$ $(\rho-l)^{2}+z^{2}$ in Eq. (25); this implies the presence of the current of quarks in the $z=0$ plane located on a circle of radius $l$. As in the case of the presence of the charge density considered above, we will change the value of the arbitrary constant $\left(j_{0}\right)^{7 \varphi}$ and keep track of the behavior of the electric and magnetic fields. As a result, it turns out that both fields may exist simultaneously only in a very restricted range of values of $\left(j_{0}\right)^{7 \varphi}$. For example, for the same fixed values of the system parameters as those used in the case with a charge, there is the following range of possible values $-11 \lesssim\left(j_{0}\right)^{7 \varphi} \lesssim 2$ for which the system possesses both the electric and magnetic fields. When one goes beyond this range, only the magnetic field remains.
Thus there are some critical values of $\left(j_{0}\right)^{7 \varphi}$ for which the electric field is switched off. One might expect that for another values of the system parameters, distinct from those for which the aforementioned range of $\left(j_{0}\right)^{7 \varphi}$ has been found, there will take place a similar situation where there will be some critical values of $\left(j_{0}\right)^{7 \varphi}$ determining the range in which configurations both with electric and magnetic fields do exist.

The corresponding field strength distributions are given in Fig. 11. It is seen from them that the qualitative picture of the electric field strength distribution changes only slightly (the same is true of the strength of the field $\vec{E}^{5}$ that is still a vortex field). In turn, the distribution of the magnetic field may depend considerably on the value of the constant $\left(j_{0}\right)^{7 \varphi}$; this especially manifests itself for large negative values of $\left(j_{0}\right)^{7 \varphi}$ when the influence of the external source of the field becomes already comparable with the contribution caused by the nonlinearity of the Proca fields. 

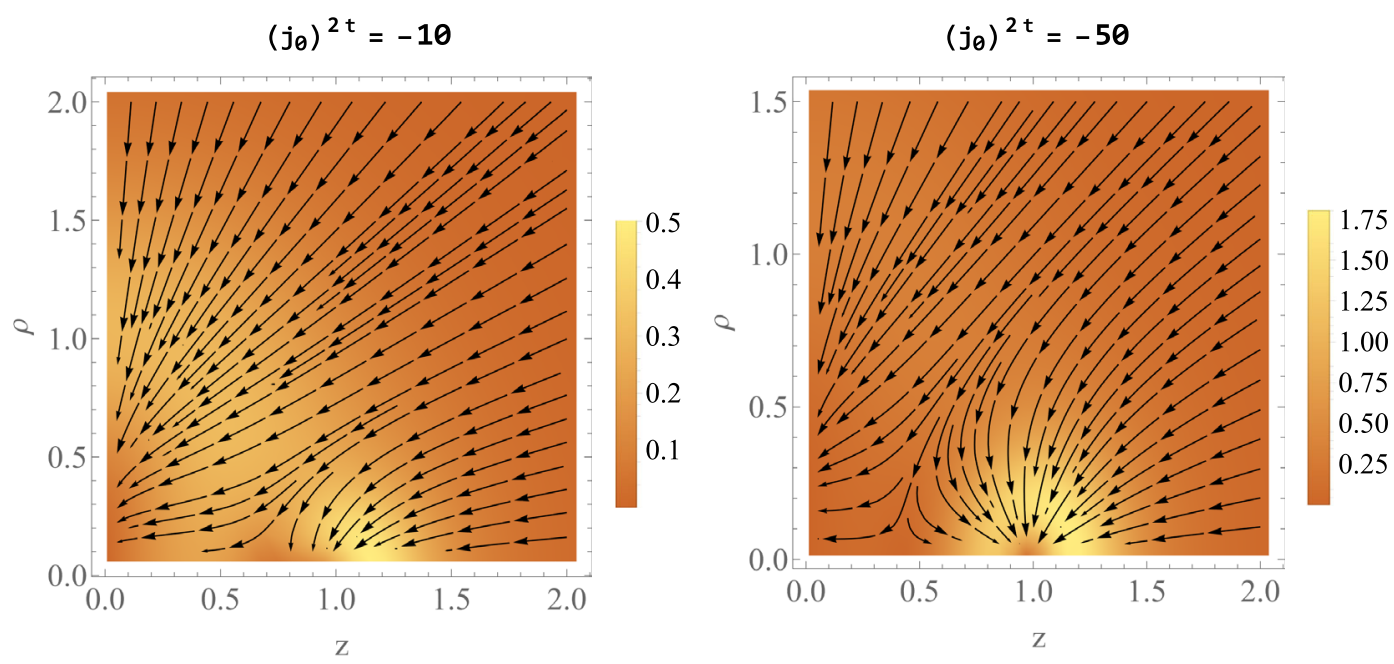

$\left(j_{\ominus}\right)^{2 t}=-300$

$\left(j_{\theta}\right)^{2 t}=-1000$


FIG. 10. The color electric field $\vec{E}^{2}$ strength distributions for different values of the parameter $\left(j_{0}\right)^{2 t}$ and fixed values $\mu_{1}=3.3$ and $\mu_{2}=3.7$. The parameters $M=1.4, \Lambda=1, \lambda=10, g=1, R_{0}=0.2$, and $l=1$.

\section{Classical stability}

Here we address briefly the question of classical stability of the configurations under investigation. In studying systems supported by various fundamental fields, one uses several stability criteria. First, this is topological stability [18]. Typical examples of configurations for which such criterion can be applied are topological (kinklike) solutions for scalar fields [whose stability is ensured by the difference in the values of the field at spatial infinities, $\phi(-\infty) \neq \phi(+\infty)]$ and the 't Hooft-Polyakov monopole solution, when, as $r \rightarrow \infty$, the scalar field approaches different limits in different directions. It is evident that since the solutions considered here are nontopological ones, this criterion is not applicable to our case.

Second, one can use a stability criterion related to the presence of some conserved quantities in a system. In particular, the question of stability of nontopological solutions in $\mathrm{SU}(2)$ theory has been considered in
Ref. [19], where a spherically symmetric system with a Higgs-type field is under investigation. There, a crucial ingredient is the presence of a conserved nonvanishing isospin: stable solutions do exist only when the total isospin exceeds some critical value. A similar situation occurs in the case of a system with two scalar fields, one of which is complex and the other is real [20]. In this case the system possesses a nonzero charge associated with the complex scalar field, and stable solutions do exist only for definite values of the charge. Since our system is supported by real fields, there is no conserved charge (or particle number), and such a stability criterion cannot be employed as well.

Another possibility is to study the classical stability with respect to linear and nonlinear perturbations of a different type. In the case of spherically symmetric configurations, such studies have been performed frequently in the literature for various systems, including investigations both of nongravitating nontopological systems (see, e.g., Ref. [21]) 



FIG. 11. The electric and magnetic fields strength distributions for different values of the parameter $\left(j_{0}\right)^{7 \varphi}$ and fixed values $\mu_{1}=3.3$ and $\mu_{2}=3.7$. The parameters $M=1.4, \Lambda=1, \lambda=10, g=1, R_{0}=0.2$, and $l=1$. For $\left(j_{0}\right)^{7 \varphi}=-15$ and 10 , the electric field is absent.

and of different objects supported by gravitating nonAbelian fields (see, e.g., the problems with linear [22] and nonlinear [23] perturbations). However, for axially symmetric systems of the type considered in the present paper, this is a much more technically complicated problem that requires a careful analysis. 
Finally, there is a possibility to examine the stability within catastrophe theory. As applied to solitons, it was used in Ref. [24]. When gravity is present, this theory was applied to boson (spin-0) spherically symmetric systems in Ref. [25] and has been extended to the case of axially symmetric nontopological soliton solutions in Ref. [26].

Keeping all this in mind and taking into account that a study of the question of stability of the axially symmetric system under consideration will require a fair amount of effort, we plan to do this in a separate work.

\section{DISCUSSION AND CONCLUSIONS}

In the present paper, we have studied particlelike solutions with the flux of a magnetic field in the nonAbelian Proca-Higgs theory. The crucial feature of these solutions is that they are localized in space and have the flux of the color magnetic Proca field passing through the central cross section of the Proca tube. This allows the possibility of comparing the field configurations obtained with the Nielsen-Olesen tube. In both cases, there are common features: (i) the presence of a longitudinal magnetic field creating the flux of the magnetic field over the transverse cross section of the tube; and (ii) the scalar field pushes out the magnetic field (the analog of the Meissner effect). The main distinctions between the Proca tube and the Nielsen-Olesen tube are: (i) the Proca tube has finite size, whereas the Nielsen-Olesen tube is infinite; and (ii) the Proca tube is a topologically trivial object.

Natural questions arising in this context are: (i) Do Proca fields really exist in nature? (ii) Where the tubes obtained by us could occur? Regarding the first question, it should be pointed out that at the present time numerous studies are carried out to find such fields in nature, see, e.g., Refs. [5,27-29]. According to another point of view, Proca theories may be not fundamental but some phenomenological approaches to other theories. For instance, one might suppose that the SU(2) Proca-Higgs theory could serve as a phenomenological description of some phenomena in SU(3) QCD. For example, this could be in the case where some degrees of freedom belonging to the subgroup $\mathrm{SU}(2) \subset \mathrm{SU}(3)$ acquire mass as a result of quantization, and such degrees of freedom are approximately described by a SU(2) Proca theory, whereas the remaining degrees of freedom are purely quantum and are approximately described by a scalar field as a condensate. Of course, such a description is approximate and is rather similar to a phenomenological description of superconductivity by the Ginzburg-Landau equation.

Regarding the second question, one may suppose that if Proca fields do exist in nature (it is unimportant whether they are fundamental or phenomenological ones), at high temperatures, in non-Abelian Proca theories, there can exist a "quark-Proca-gluon-Higgs" plasma; this is analogous to what happens in QCD when the quark-gluon plasma occurs.

Summarizing the results obtained in the present paper:

(i) We have studied finite-size tube solutions in the nonAbelian Proca-Higgs theory both with and without external sources.

(ii) It is shown that such configurations have the finite total energy and the flux of the magnetic field over the transverse cross section.

(iii) It is demonstrated that there exist some critical values of the system parameters $\mu_{2}$ and $\left(j_{0}\right)^{7 \varphi}$ for which the electric field is switched off and only the magnetic field remains nonzero.

(iv) It is established that for the Proca tubes to exist, the presence of a scalar field is necessary; such field acts as Cooper pairs in a superconductor and pushes out the Proca field creating localized objects.

(v) Comparison of properties of the solutions obtained with the properties of the Nielsen-Olesen tube has been carried out.

(vi) It is assumed that such particlelike solutions may describe quasiparticles in the hypothetical "quarkProca-gluon-Higgs" plasma.

In conclusion, we would like to emphasize the rich internal structure of Proca theories allowing the possibility of obtaining the particlelike solutions studied in the present paper, whose existence is ensured by the presence of a mass of the vector Proca bosons. This is also applied to the case of strongly gravitating particlelike configurations consisting of coupled Proca and Higgs fields considered by us in Ref. [30]. For such systems, it is possible to obtain localized regular asymptotically flat solutions describing Proca-Higgs mini-boson stars possessing an axially symmetric dipole field. This in turn permits one to expect that it will also be possible to get solutions for systems containing both other components of the Proca field and another types of fundamental fields (for example, spinor ones).

\section{ACKNOWLEDGMENTS}

This research has been funded by the Science Committee of the Ministry of Education and Science of the Republic of Kazakhstan (Grant No. BR10965191 "Complex research in nuclear and radiation physics, high-energy physics and cosmology for development of the competitive technologies"). We are also grateful to the Research Group Linkage Programme of the Alexander von Humboldt Foundation for the support of this research. We thank Jutta Kunz and Burkhard Kleihaus for many useful discussions and helpful correspondence. 
[1] R. Brito, V. Cardoso, C. A. R. Herdeiro, and E. Radu, Proca stars: Gravitating Bose-Einstein condensates of massive spin 1 particles, Phys. Lett. B 752, 291 (2016).

[2] C. A. R. Herdeiro, A. M. Pombo, and E. Radu, Asymptotically flat scalar, Dirac and Proca stars: Discrete vs continuous families of solutions, Phys. Lett. B 773, 654 (2017).

[3] V. Dzhunushaliev and V. Folomeev, Dirac star in the presence of Maxwell and Proca fields, Phys. Rev. D 99, 104066 (2019).

[4] C. Herdeiro, I. Perapechka, E. Radu, and Y. Shnir, Asymptotically flat spinning scalar, Dirac and Proca stars, Phys. Lett. B 797, 134845 (2019).

[5] J. C. Bustillo, N. Sanchis-Gual, A. Torres-Forné, J. A. Font, A. Vajpeyi, R. Smith, C. Herdeiro, E. Radu, and S. H. W. Leong, GW190521 as a Merger of Proca Stars: A Potential New Vector Boson of $8.7 \times 10^{-13} \mathrm{eV}$, Phys. Rev. Lett. 126, 081101 (2021).

[6] L. Heisenberg, R. Kase, M. Minamitsuji, and S. Tsujikawa, Hairy black-hole solutions in generalized Proca theories, Phys. Rev. D 96, 084049 (2017).

[7] E. Allys, P. Peter, and Y. Rodriguez, Generalized Proca action for an Abelian vector field, J. Cosmol. Astropart. Phys. 02 (2016) 004.

[8] E. Allys, P. Peter, and Y. Rodriguez, Generalized SU(2) Proca theory, Phys. Rev. D 94, 084041 (2016).

[9] M. Minamitsuji, Solutions in the generalized Proca theory with the nonminimal coupling to the Einstein tensor, Phys. Rev. D 94, 084039 (2016).

[10] A. De Felice, L. Heisenberg, R. Kase, S. Mukohyama, S. Tsujikawa, and Y.1. Zhang, Cosmology in generalized Proca theories, J. Cosmol. Astropart. Phys. 06 (2016) 048.

[11] V. Dzhunushaliev and V. Folomeev, Proca tubes with the flux of the longitudinal chromoelectric field and the energy flux/momentum density, Eur. Phys. J. C 80, 1043 (2020).

[12] V. Dzhunushaliev, V. Folomeev, T. Kozhamkulov, A. Makhmudov, and T. Ramazanov, Non-Abelian Proca theories with extra fields: Particlelike and flux tube solutions, Phys. Scr. 95, 074013 (2020).

[13] V. Dzhunushaliev, V. Folomeev, and A. Tlemisov, Linear energy density and the flux of an electric field in Proca tubes, Symmetry 13, 640 (2021).

[14] H. B. Nielsen and P. Olesen, Vortex-line models for dual strings, Nucl. Phys. B61, 45 (1973).
[15] E. V. Shuryak and I. Zahed, Rethinking the properties of the quark gluon plasma at T approximately T(c), Phys. Rev. C 70, 021901 (2004).

[16] E. V. Shuryak and I. Zahed, Towards a theory of binary bound states in the quark gluon plasma, Phys. Rev. D 70, 054507 (2004).

[17] W. Schönauer and R. Weiß, Efficient vectorizable PDE solvers, J. Comput. Appl. Math. 27, 279 (1989).

[18] R. Rajaraman, An Introduction to Solitons and Instantons in Quantum Field Theory (North-Holland, Amsterdam, 1982).

[19] R. Friedberg, T. D. Lee, and A. Sirlin, Gauge field nontopological solitons in three space dimensions. 1., Nucl. Phys. B115, 1 (1976).

[20] R. Friedberg, T. D. Lee, and A. Sirlin, A class of scalar-field soliton solutions in three space dimensions, Phys. Rev. D 13, 2739 (1976).

[21] T. D. Lee and Y. Pang, Nontopological solitons, Phys. Rep. 221, 251 (1992).

[22] N. Straumann and Z. H. Zhou, Instability of the BartnikMckinnon solution of the Einstein Yang-Mills equations, Phys. Lett. B 237, 353 (1990).

[23] Z.h. Zhou and N. Straumann, Nonlinear perturbations of Einstein Yang-Mills solitons and non-Abelian black holes, Nucl. Phys. B360, 180 (1991).

[24] F. V. Kusmartsev, Application of catastrophe theory to molecules and solitons, Phys. Rep. 183, 1 (1989).

[25] F. V. Kusmartsev, E. W. Mielke, and F. E. Schunck, Gravitational stability of boson stars, Phys. Rev. D 43, 3895 (1991).

[26] B. Kleihaus, J. Kunz, and S. Schneider, Stable phases of boson stars, Phys. Rev. D 85, 024045 (2012).

[27] R. Kase, M. Minamitsuji, and S. Tsujikawa, Neutron stars with a generalized Proca hair and spontaneous vectorization, Phys. Rev. D 102, 024067 (2020).

[28] N. Sanchis-Gual, C. Herdeiro, J. A. Font, E. Radu, and F. Di Giovanni, Head-on collisions and orbital mergers of Proca stars, Phys. Rev. D 99, 024017 (2019).

[29] A. de Felice, L. Heisenberg, and S. Tsujikawa, Observational constraints on generalized Proca theories, Phys. Rev. D 95, 123540 (2017).

[30] V. Dzhunushaliev and V. Folomeev, Axially symmetric Proca-Higgs boson stars, Phys. Rev. D 104, 104024 (2021). 